\title{
Presente e futuro na pesquisa em nutrição renal
}

\section{Present and future in renal nutrition research}

\author{
Entrevistado: Juan Jesus Carrero ${ }^{1}$ \\ Entrevista conduzida por: Carla Maria Avesani ${ }^{2}$
}

\begin{abstract}
1 Em março de 2012, Juan Jesus Carrero visitou 0 Departamento de Nutrição Aplicada / Instituto de Nutrição - UERJ, como Professor Convidado.

${ }^{2}$ Carla Maria Avesani é nutricionista clínica e pesquisadora especializada em aspectos nutricionais da DRC. Seu estudo de doutorado foi realizado na Universidade Federal de São Paulo. Depois disso, passou 12 meses na Karolinska Institute onde fez seu pós-doutorado em nutrição em doença renal crônica no grupo de pesquisa liderado pelo Dr. Bengt Lindholm e Dr. Peter Stenvinkel. Nesse período trabalhou com Juan Jesus Carrero. Atualmente é Professora Adjunta do Departamento de Nutrição Aplicada / Instituto de Nutrição - UERJ. É coordenadora do grupo de pesquisa que estuda os aspectos nutricionais de pacientes idosos em hemodiálise do Instituto de Nutrição - UERJ, e nutricionista do ambulatório de tratamento conservador do Hospital Universitário Pedro Ernesto.

Esta entrevista Foi traduzida para o português por Fernanda Galvão, mestranda do curso de Pós-Graduaç̦ão em Alimentação, Nutrição e Saude (PPG-ANS) do Instituto de Nutrição dá UERJ.
\end{abstract}

Juan Jesus Carrero foi graduado em farmácia pela Universidade Granada (Espanha), e dedicou seus estudos e pesquisas desde então ao campo da nutrição. Seu estudo para obtenção do título de PhD na Universidade de Granada, Espanha, foi versado sobre estratégias de suplementação nutricional em populações com alto risco cardiovascular. Vários estudos experimentais randomizados controlados testaram os efeitos da suplementação nutricional com ácidos graxos w3, ácido oléico e vitaminas B6, B9 e E. Durante seu pós-doutorado ingressou no Departamento de Medicina Renal no Instituto Karolinska, na Suécia, onde se aprofundou no campo dos aspectos nutricionais da doença renal crônica (DRC), com ênfase na associação entre desnutrição energético protéica (DEP), inflamação e doença cardiovascular (DCV). Dentro deste contexto, ele alcançou o segundo grau de $\mathrm{PhD}$ na Universidade Karolinska. Ele então desenvolveu sua linha de pesquisa no Instituto Karolinska (Karolinska Institutet) nas áreas de distúrbios de composição corporal, deficiências nutricionais, distúrbios endócrinos e consequências da inflamação sistêmica na DRC. Ele já alconcou a marca de mais de 120 publicações 
nas melhores revistas de nefrologia e, em minha opinião, é atualmente um dos mais promissores jovens pesquisadores no campo da nutrição e DRC. Em março de 2012, Juan Jesus Carrero visitou o Departamento de Nutrição Aplicada / Instituto de Nutrição - Universidade Estadual do Rio de Janeiro - como Professor Visitante (com bolsa concedida pela Subreitoria de Pós-graduação e Pesquisa (SR2) da UERJ). Sua visita permitiu uma importante interação com os alunos de pós-graduação do Programa de Nutrição, Saúde e Alimentação (Mestrado e Doutorado) e discutiu vividamente sua área de especialização e uso adequado de epidemiologia/estatística na pesquisa. Esta entrevista tem como objetivo partilhar a sua opinião sobre as direções atuais e futuras na pesquisa no campo de nutrição e doença renal crônica.

Demetra: Por muitos anos, a desnutrição foi o distúrbio nutricional mais discutido no campo da nutrição e DRC. No entanto, após a publicação da epidemiologia reversa da obesidade nos pacientes em hemodiálise (HD) em 2003 (Kalantar-Zadeh et al, Kidney Int 63:793, 2003), iniciou-se uma mudança na direção do papel da obesidade na sobrevivência de pacientes com DRC (em ambos estágios, pré-diálise e diálise). Muitos estudos têm sido publicados sobre o assunto. Com isto em mente, qual é a sua opinião sobre o papel da obesidade como fator protetor ou não protetor sobre a mortalidade na população?

Juan Jesus Carrero: Penso que ambos os conceitos não são opostos, mas complementares. Desnutrição/ "depleção ou emaciamento" é uma condição tremendamente devastadora e com rápido impacto sobre a composição corporal e sobrevida do paciente. No contexto de uma desnutrição acelerada, indivíduos obesos têm capacidade de sobrevivência ligeiramente mais longa devido a maiores depósitos de energia disponíveis para o hipercatabolismo corporal. Neste contexto, a obesidade é "protetora" a curto prazo, uma vez que permite aos pacientes com desnutrição energético protéica resistir mais. No entanto, a longo prazo, a obesidade é um fator de risco associado à hipertensão, diabetes, aterosclerose e inflamação.

Demetra: Este assunto também traz antecipadamente as novas descobertas dos distúrbios metabólicos provenientes da obesidade, o estágio de disfunção metabólica levando à resistência à insulina, inflamação de baixo grau (ou inflamação subclínica), hipertrigliceridemia, e todos juntos aumentam a chance de DCV. Como se pode equilibrar estas descobertas com as maiores taxas de sobrevida encontradas nos pacientes em HD com maior índice de massa muscular (IMC)?

Juan Jesus Carrero: Como eu disse é uma questão de riscos competitivos. Desnutrição/ "depleção ou emaciamento" é um dos riscos de curto prazo, que compete com os problemas da obesidade. Nesta competição, a desnutrição vence e o paciente morre por desnutrição. Quando desnutrição não está presente, o paciente pode morrer de distúrbios metabólicos associados à obesidade. 
Demetra: Métodos de composição corporal também são interessantes quando se discute a obesidade em pacientes com DRC. Para fins de pesquisa e clínicos, qual o método que você recomendaria para avaliar a composição corporal de pacientes com DRC?

Juan Jesus Carrero: IMC tem problemas devido à incapacidade em diferenciar tecido muscular de tecido adiposo e em relação a questão de hiper-hidratação no contexto da DRC. Eu acho que a circunferência da cintura é uma estimativa fácil e confiável de gordura abdominal e a força de preensão manual é uma estimativa válida da força muscular. Há, naturalmente, avaliações muito melhores, mas como uma ferramenta de triagem na clínica estes são suficientemente válidos.

Demetra: Interessante também, é a constatação de que os doentes obesos também podem ter sinais de desnutrição protéica, caracterizada por um estado de sarcopenia. A coexistência de obesidade e sarcopenia foi definida por alguns como sarcopenia da obesidade. Em pacientes com DRC não há muitos estudos publicados até agora sobre este assunto. Você pode nos dizer antecipadamente qual é o seu palpite sobre esta condição clínica tão particular?

Juan Jesus Carrero: Na verdade, esta questão nunca foi estudada em pacientes com DRC. Um estudo pioneiro avaliou a prevalência de desnutrição no contexto da obesidade (Honda et al. Am J Clin Nutr 86: 633, 2007). No entanto, a avaliação da sarcopenia da obesidade ainda é precária. Perda de massa muscular é uma condição frequente em nossos pacientes e é melhor indicador de resultado em termos de ferramentas de avaliação da composição corporal. Sobrepeso, obesidade e hiper-hidratação podem esconder a visualização da atrofia muscular e, provavelmente, sub diagnosticá-la. Acho que este é um aspecto fascinante e com potencial clinico relevante da DEP urêmica, especialmente tendo em conta o crescimento da epidemia da obesidade na população DRC.

Demetra: Como a sarcopenia poderia ser tratada? Você tem conhecimento de estudos clínicos com o objetivo de tratar a sarcopenia (independentemente de ser em obesos ou não) em pacientes com DRC?

Juan Jesus Carrero: O tratamento dependerá das causas da sarcopenia nesta população. Catabolismo muscular na DRC é multifatorial e resumidamente, ocorre principalmente como consequência da inflamação persistente, pela falta deficiência aminoácidos a partir da dieta, pela acidose metabólica e pela resistência à insulina ao GH/IGF-1, deficiência de testosterona e etc. Há tratamentos disponíveis para cada uma dessas condições, mas poucos têm sido devidamente testados em pacientes urêmicos até o momento.

Demetra: A desnutrição, recentemente definida como "depleção ou emaciamento" energético protéica também é de grande importância devido à sua estreita associação com as taxas de mortalidade crescentes. Como podemos diagnosticar a "depleção" energético-protéica (ou avaliar) em pacientes com DRC?

Juan Jesus Carrero: O diagnóstico é difícil porque atualmente não há consenso sobre sua definição. A Sociedade Internacional de Nutrição e Metabolismo Renal propôs alguns critérios de diagnóstico, mas estes ainda não foram validados. De amneira geral, recomenda-se mepregar um conjunto de métodos, sendo 
esses, o cálculo de ingestão alimentar, a estimativa da composição corporal, o uso de métodos compostos de avaliação do estado nutricional e de parâmetros laboratoriais, tais como albumina de sérica são utilizados para diagnosticar DEP e para avaliar o grau de gravidade da DEP sem validar claramente os critérios de diagnóstico. Um debate importante é se a concentrações séricas de albumina seria um marcador do estado nutricional, de inflamação, de comorbidade, ou ainda de outras condições. A não definição até o momento do significado clínico da reduzida concentração d elabumina sérica levou à mensagens “confusas”ou pouco claras quanto ao diagnóstico tratamento da DEP na DRC. Independentemente da causa da hipoalbuminemia em pacientes em diálise, as evidências sugerem que as intervenções nutricionais podem aumentar a albumina sérica em pacientes em diálise. Por isso, devemos continuar avaliando a albumina sérica e outros parâmetros substitutos do estado nutricional para a estratificação do risco dos pacientes e para alocar a terapia nutricional, enquanto estudos, bem concebidos, em grande escala, randomizados e controlados, sobre os efeitos da ingestão nutricional em resultados clínicos sejam realizados.

Uma consideração é que talvez seja difícil ter critérios rigorosos para avliacao do estado nutricional na DRC que sejam aplicáveis a todos os pacientes, sociedades e culturas. Além disso, características das amostras dos estudos podem variar com a idade e com o contexto da doença. Por exemplo, o mesmo ponto de corte para diagnosticar hipoalbuminemia em idosos pode não ser o mesmo daquele empregado em pacientes jovens. Além disso, o valor de albumina sérica para diagnóstico da hipoalbuminemia em pacientes em diálise peritoneal provavelmente é menor do que para os pacientes submetidos em hemodiálise. A fim de tomar uma decisão sobre diagnóstico/avaliação da DEP, não podemos depender exclusivamente desses biomarcadores. Precisamos equilibrar o auto-relato dos pacientes, o julgamento clínico e as informações obtidas a partir destes instrumentos de avaliação.

Demetra: Estudos clínicos que tentaram tratar a DEP não conseguiram reverter essa condição. Por que é tão difícil de tratar a DEP?

Juan Jesus Carrero: A principal dificuldade começa com o fato de que verdadeiramente é uma síndrome multifatorial, em que a desnutrição pura convive com a inflamação/hipercatabolismo, e resistência aos hormônios anabólicos, além de e um estilo de vida sedentário. As estratégias de tratamento até agora têm se concentrado em atingir somente um desses componentes. $\mathrm{O}$ tratamento adequado de uma síndrome multifatorial como esta, provavelmente deve ser multifacetado e combinar suporte nutricional com antiinflamatórios estratégicos, treinamento físico e hormônios anabolizantes.

\section{Demetra: Neste campo adversário: Obesidade versus DEP, que distúrbio nutricional tem o pior resultado e menor qualidade de vida? Qual, na sua opinião, deve ser o primeiro alvo nos projetos de pesquisa?}

Juan Jesus Carrero: DEP, sem dúvida. DEP também existe em pessoas obesas, e é mais difícil diagnosticála nestes indivíduos, pois interpretamos seu excesso de peso como um sinal de saúde. Quando se torna evidente, pode ser tarde demais. Portanto, a avaliação nutricional é necessária e exigida frequentemente em todos os pacientes com DRC, independentemente do seu IMC. 